$17^{\text {th }}$ International Congress of Metrology, 10003 (2015)

DOI: $10.1051 /$ metrology / 201510003

(C) Owned by the authors, published by EDP Sciences, 2015

\title{
A calibration system for electric power at frequencies up to $1 \mathrm{MHz}$
}

\author{
Tobias Bergsten ${ }^{1}$, Valter Tarasso ${ }^{1}$ and Karl-Erik Rydler ${ }^{1}$ \\ ${ }^{1}$ SP Technical Research Institute of Sweden, Box 852 SE-50115 Borås, Sweden
}

\section{Introduction}

Electrical power measurements are a central part of society today. Traditional power measurements have been focused on the power delivered by the $50 \mathrm{~Hz}$ or $60 \mathrm{~Hz}$ electrical grid with up to about 50 harmonics. This means that the bandwidth of such a power meter only needs to be a few $\mathrm{kHz}$.

Most modern electrical devices and machines are powered by batteries, or by the grid via DC converters. Ironically, measuring power in these devices requires higher bandwidth, because most modern devices use switching power converters, either to convert AC grid power to DC power, or to convert between different DC voltage levels. These power converters work at switching frequencies of several $\mathrm{kHz}$ up to several tens of $\mathrm{kHz}$. Higher frequencies means potentially lower power losses, so the trend is towards higher frequencies.

Many instrument manufacturers now offer power meters with bandwidth in the $\mathrm{MHz}$ range. In order to address the calibration needs for these instruments, we have developed a reference power calibration system for frequencies up to $1 \mathrm{MHz}$. In this article we describe the system and the procedures for ensuring traceable power measurements.

\section{Measurement setup}

Figure 1 illustrates the components which make up the system. The colours indicate the voltage channel at the top (blue), the current channel at the bottom (green) and the device under test, DUT (pink).

The phase between the current and voltage signals is controlled by two arbitrary waveform generators, AWG (National Instruments NI PXI-5421/5441) which provide synchronised sine waves to the phase lock inputs of the AC sources (Fluke 5700A).

The AC source for the current channel may output current directly, or it may provide the input voltage signal to a transconductance amplifier (Clarke-Hess 8100). The current is connected to a current shunt (SP design) in series with the current input of the power meter DUT. The voltage of the shunt is measured differentially with a two channel digitizer (National Instruments NI PXI5922).

The AC source for the voltage channel will output the voltage directly, or provide the input voltage for a voltage amplifier (Fluke 5205). The voltage signal is connected in parallel to the voltage input of the DUT, and to a resistive voltage divider (SP design) with a buffer amplifier (NMIA/SP design) on the output. The output signal from the buffer is measured differentially with a second digitizer card, synchronised with the first.

During calibration of the DUT the phase between the current and voltage signals can be unpredictable at higher frequencies, because of load and frequency dependence of the AC sources. Therefore the phase is first measured by the digitizers and the AWG phase shift adjusted accordingly to get the desired phase. Then the calibration

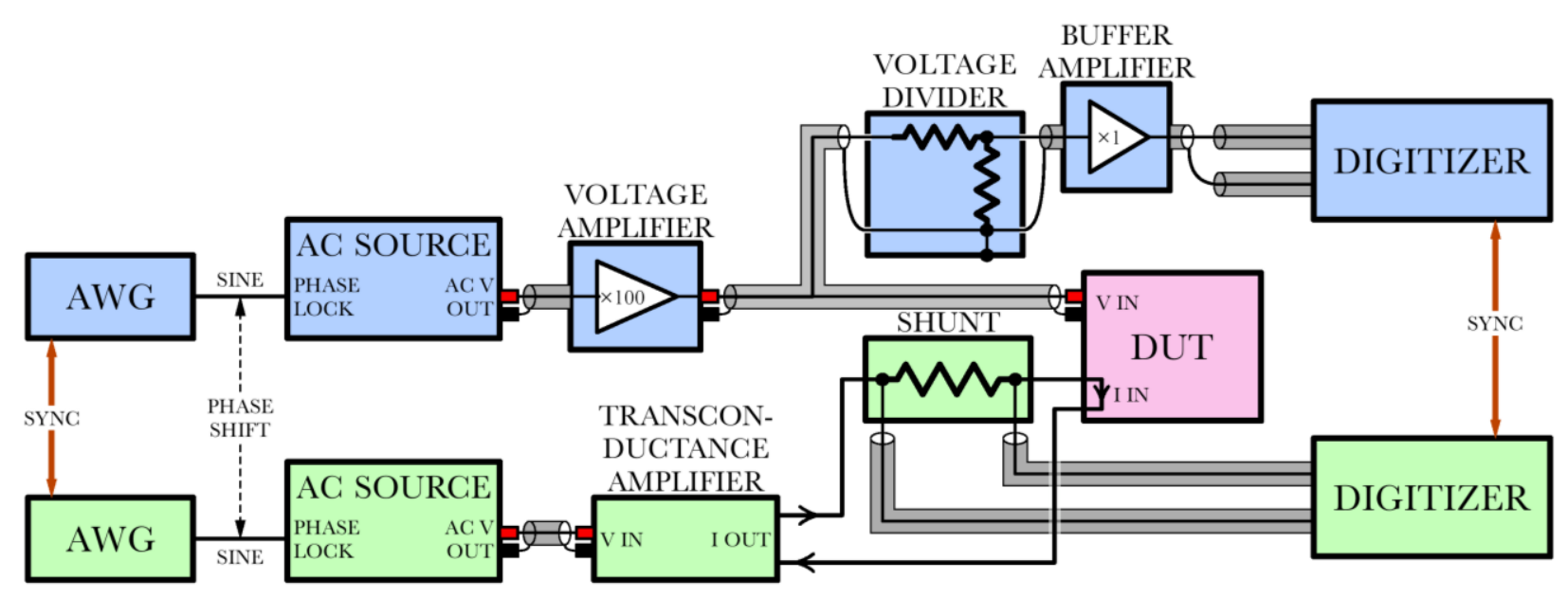

Figure 1. Schematic picture of the system components and the device under test (DUT). 


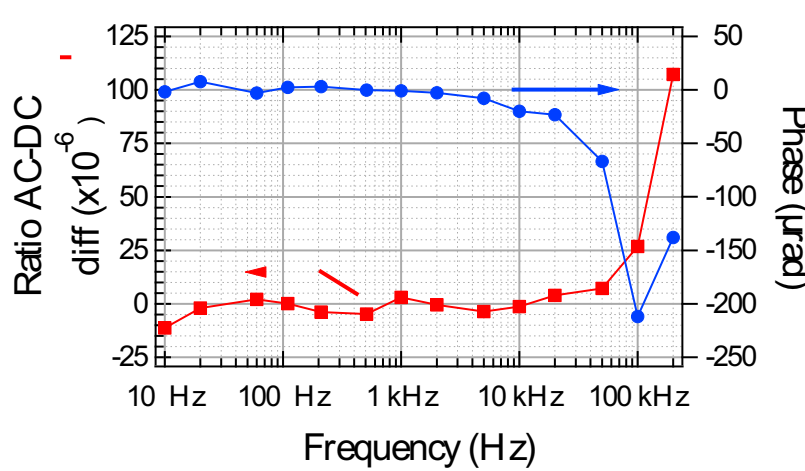

Figure 2. Typical result of AC-DC difference and phase response calibration of a voltage divider (ratio 150:1, $120 \mathrm{~V}$ nominal input voltage).

measurement takes place with the digitizers and the DUT measuring the power simultaneously.

\section{Calibration of the system components}

In order to provide traceable power measurements with this system, we calibrate the critical components of the system separately. The voltage dividers are calibrated for amplitude ratio and phase response, in combination with the buffer amplifier. The shunts are calibrated for complex impedance. The digitizers are calibrated for amplitude response, phase shift between the channels, and input impedance.

\subsection{Voltage dividers}

The calibration of the voltage dividers consist of three parts: DC ratio, AC-DC difference and phase response. We calibrate the combination of passive divider plus buffer amplifier in all cases and the input is defined as the end of a $1 \mathrm{~m}$ coaxial cable (RG58) connected to the divider input terminals. The DC ratio is a standard measurement which is performed using a high accuracy voltmeter.

The AC-DC difference and the phase response calibrations are performed in a stepping procedure using the digitizers. First the lowest ratio divider (5:1) is measured (ratio and phase) by comparing the input and output signals. Then we connect the next higher ratio divider in parallel with the first and measure the output ratios. We use the low frequency points below $100 \mathrm{~Hz}$ as the effective DC value and calculate the AC-DC difference. We can do this because we know from regular AC-DC difference measurements that the values below $100 \mathrm{~Hz}$ are below the noise level.

We get also the phase response in the same stepping procedure. However, we also need to make corrections for voltage level and power dependence for the higher ratio dividers. This is described in ref. [1]. A typical calibration result is shown in Fig. 2.

\subsection{Current shunts}

The procedure for calibrating current shunts is similar to the voltage divider procedure. The complex impedance ratios are measured by connecting two shunts in series and measuring the output signals using digitizers. We

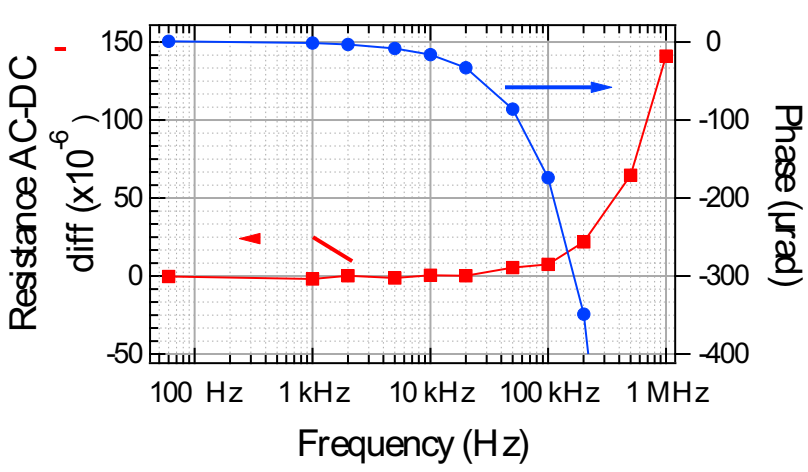

Figure 3. Typical result of AC-DC difference and phase response calibration of a current shunt (resistance $0.4 \Omega, 2 \mathrm{~A}$ nominal input current).

need to take into account the input impedance of the digitizer channels measuring between the shunts, because they will draw a small amount of current. Therefore we switch the two shunts and measure again, in order to cancel the leakage current effect.

In order to have a reference for the phase we use a set of three shunts which are geometrically identical, but with different resistance values. Using the procedure described in [2] we can determine the inductance and capacitance of the shunts, and we can calculate the phase of the shunt inductance. Figure 3 gives an example of the result.

\subsection{Digitizer calibration}

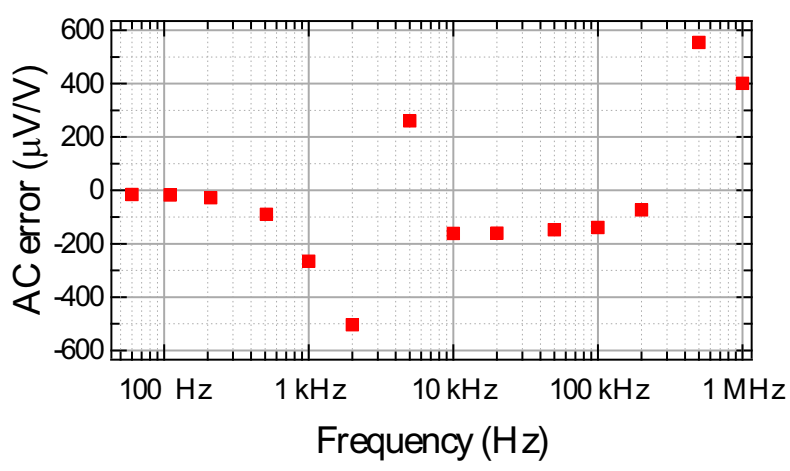

Figure 4. Typical result of AC frequency response calibration of a digitizer ( $\pm 5 \mathrm{~V}$ range).

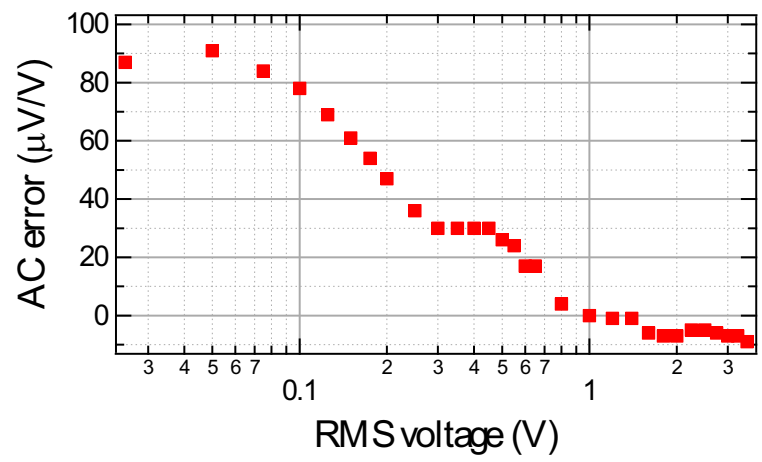

Figure 5. Typical result of AC voltage response calibration of a digitizer ( $\pm 5 \mathrm{~V}$ range). 


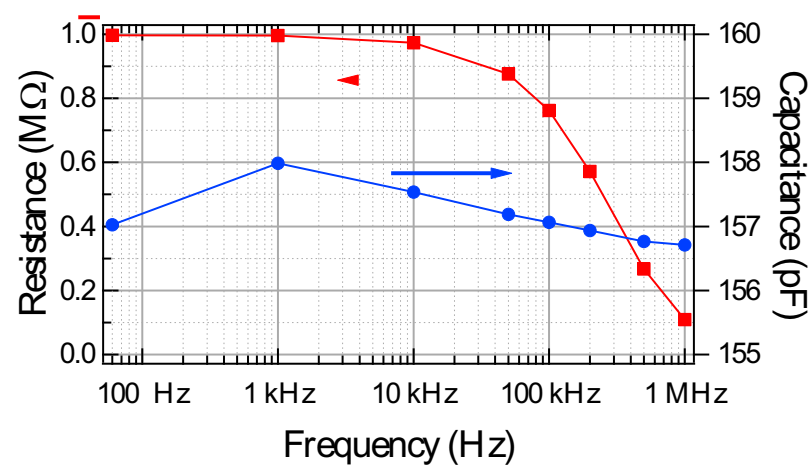

Figure 6. Typical result of input impedance calibration of a digitizer with $1 \mathrm{~m}$ coaxial cable (RG58) connected to the input terminal.

The digitizer channels need to be calibrated for amplitude response and for relative phase difference between the channels. Like the dividers, the digitizer input is defined as the end of a $1 \mathrm{~m}$ coaxial cable connected to the input terminal. This definition is particularly important at higher frequencies (above 100 $\mathrm{kHz}$ ) to take into account standing waves caused by reflections at the high impedance digitizer input.

To calibrate amplitude response, we use a thermal transfer standard, TTS (Fluke 792A) and a high performance multimeter (Fluke 8508A) to measure the $\mathrm{AC}$ response of one digitizer channel via AC-DC difference. We measure both at constant voltage $(1 \mathrm{~V}$ rms) and at constant frequency $(1 \mathrm{kHz})$. We use a variable sampling rate set to five times the signal frequency, down to the lowest supported rate of 50 ksamples/s. The amplitude error varies quite considerably with frequency (see Fig. 4); at low frequencies because of the digital filter used in the digitizer, and at high frequencies because of standing wave effects in the cable. But the stability of this amplitude response is very good, less than $10^{-5}$ except at the highest frequencies above 200 $\mathrm{kHz}$.

The voltage non-linearity is important as well. The signal levels measured varies with the current and voltage, and as Fig. 5 demonstrates, the error can vary by more than $100 \mu \mathrm{V} / \mathrm{V}$ for different amplitudes. We normalize the voltage level calibration at $1 \mathrm{~V} \mathrm{rms}$, and use it as an additional correction. The level dependence is quite independent of frequency, so we only need to do this at one frequency.

The AC-DC calibration is only performed on one digitizer channel. In order to calibrate the other channels, we perform a relative measurement by connecting all four

Table 1. An example of the results of a calibration of a power analyzer (Yokogawa WT3000). The table shows the correction factors in $\%$ for power measurements at different frequencies and current ranges (voltage range is $100 \mathrm{~V}$ ).

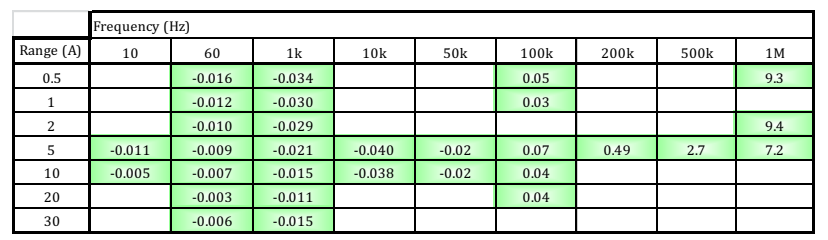

Table 2. The CMCs of SP for power measurements using the high frequency power measurement system.

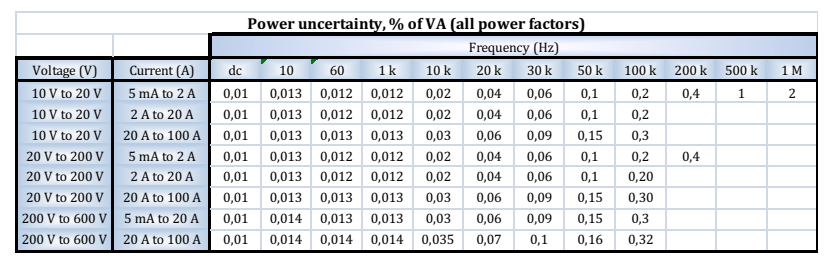

channels in parallel and measuring the difference in amplitude and phase between the channels at different frequencies and voltage levels. By combining the relative and the absolute AC-DC measurement we get an absolute calibration of all channels.

Finally we also calibrate the input impedance of the digitizer channel which measures the voltage between the shunt and the DUT, in order to correct for the current which does not enter the DUT. This impedance includes the $1 \mathrm{~m}$ cable connected to the digitizer terminal. Figure 6 shows a typical result, with an almost constant capacitance, and a strongly frequency dependent resistance. This calibration is most important at low current and for the phase at high frequency.

\section{System performance}

A LabVIEW program is used to control all the instruments in the setup, including the DUT. The calibration of a DUT can be automated using a simple script, but voltage dividers and current shunts need to be manually connected to match the voltage and current levels. The calibration values for dividers, shunts and digitizers are integrated in the program and are used to calculate the measured reference power. The DUT and the reference system measures the power simultaneously and the DUT correction values are calculated and collected in a table such at table 1 .

SP has published CMCs (Calibration and Measurement Capabilities) for power measurements up to $1 \mathrm{MHz}$. The uncertainty values for different ranges and frequencies are shown in table 2. Although we have not performed any international comparison yet, we have verified it at low frequency by comparing it with our low frequency power reference system. We calibrated a power analyser at $60 \mathrm{~Hz}$ and $1 \mathrm{kHz}(100 \mathrm{~V}, 5 \mathrm{~A})$ with both systems, and the result agreed within $3 \mu \mathrm{V} / \mathrm{V}$.

This system is also used to calibrate current transducers, such as zero-flux current transformers or current shunts, for amplitude and phase response. We use a calibrated shunt as a reference and measure it with one digitizer, and we measure the output of the transducer with the other digitizer (directly or via another shunt or voltage divider).

\section{References}

[1] Bergsten, T.; Tarasso, V.; Rydler, K.-E., "Determining voltage dependence of the phase response in voltage dividers," Precision Electromagnetic Measurements (CPEM), 2012 Conference on , vol., no., pp.282,283, 1-6 July 2012 


\section{Web of Conferences}

[2] Rydler, K.-E.; Bergsten, T.; Tarasso, V., "Determination of phase angle errors of current shunts for wideband power measurement," Precision Electromagnetic Measurements (CPEM), 2012 Conference on , vol., no., pp.284,285, 1-6 July 2012 